Algebraic $\& \mathcal{G}$ Geometric Topology

Volume 3 (2003) 905-920

Published: 29 September 2003

ATG

\title{
On the slice genus of links
}

\author{
VINCENT FLORENS \\ PATRICK M. GILMER
}

\begin{abstract}
We define Casson-Gordon $\sigma$-invariants for links and give a lower bound of the slice genus of a link in terms of these invariants. We study as an example a family of two component links of genus $h$ and show that their slice genus is $h$, whereas the Murasugi-Tristram inequality does not obstruct this link from bounding an annulus in the 4-ball.
\end{abstract}

AMS Classification 57M25; 57M27

Keywords Casson-Gordon invariants, link signatures

\section{Introduction}

A knot in $S^{3}$ is slice if it bounds a smooth 2-disk in the 4-ball $B^{4}$. Levine showed [Le] that a slice knot is algebraically slice, i.e. any Seifert form of a slice knot is metabolic. In this case, the Tristram-Levine signatures at the prime power order roots of unity of a slice knot must be zero. Levine showed also that the converse holds in high odd dimensions, i.e. any algebraically slice knot is slice. This is false in dimension 3: Casson and Gordon [CG1, CG2, G] showed that certain two-bridge knots in $S^{3}$, which are algebraically slice, are not slice knots. For this purpose, they defined several knot and 3-manifold invariants, closely related to the Tristram-Levine signatures of associated links. Further methods to calculate these invariants were developed by Gilmer [Gi3, Gi4], Litherland [Li], Gilmer-Livingston [GL], and Naik [N]. Lines [L] also computed some of these invariants for some fibered knots, which are algebraically slice but not slice. The slice genus of a link is the minimal genus for a smooth oriented connected surface properly embedded in $B^{4}$ with boundary the given link.

The Murasugi-Tristram inequality (see Theorem 2.1 below) gives a lower bound on the slice genus of a link in terms of the link's Tristram-Levine signatures and related nullity invariants. The second author [Gi1] used Casson-Gordon invariants to give another lower bound on the slice genus of a knot. In particular 
he gave examples of algebraically slice knots whose slice genus is arbitrarily large. We apply these methods to restrict the slice genus of a link.

We study as an example a family of two component links, which have genus $h$ Seifert surfaces. Using Theorem 4.1, we show that these links cannot bound a smoothly embedded surface in $B^{4}$ with genus lower than $h$, while the MurasugiTristram inequality does not show this. In fact there are some links with the same Seifert form that bound annuli in $B^{4}$. We work in the smooth category.

The second author was partially supported by NSF-DMS-0203486.

\section{Preliminaries}

\subsection{The Tristram-Levine signatures}

Let $L$ be an oriented link in $S^{3}$, with $\mu$ components, and $\theta_{S}$ be the Seifert pairing corresponding to a connected Seifert surface $S$ of the link. For any complex number $\lambda$ with $|\lambda|=1$, one considers the hermitian form $\theta_{S}^{\lambda}:=(1-$ ג) $\theta_{S}+(1-\bar{\lambda})\left(\theta_{S}\right)^{T}$. The Tristram signature $\sigma_{L}(\lambda)$ and nullity $n_{L}(\lambda)$ of $L$ are defined as the signature and nullity of $\theta_{S}^{\lambda}$. Levine defined these same signatures for knots [Le]. The Alexander polynomial of $L$ is $\Delta_{L}(t):=\operatorname{Det}\left(\theta_{S}-t\left(\theta_{S}\right)^{T}\right)$. As is well-known, $\sigma_{L}$ is a locally constant map on the complement in $S^{1}$ of the roots of $\Delta_{L}$ and $n_{L}$ is zero on this complement. If $\Delta_{L}=0$, it is still true that the signature and nullity are locally constant functions on the complement of some finite collection of points.

The Murasugi-Tristram inequality allows one to estimate the slice genus of $L$, in terms of the values of $\sigma_{L}(\lambda)$ and $n_{L}(\lambda)$.

Theorem 2.1 [M, T] Suppose that $L$ is the boundary of a properly embedded connected oriented surface $F$ of genus $g$ in $B^{4}$. Then, if $\lambda$ is a prime power order root of unity, we have

$$
\left|\sigma_{L}(\lambda)\right|+n_{L}(\lambda) \leq 2 g+\mu-1 .
$$

\subsection{The Casson-Gordon $\sigma$-invariant}

In this section, for the reader convenience, we review the definition and some of the properties of the simplest kind of Casson-Gordon invariant. It is a reformulation of the Atiyah-Singer $\alpha$-invariant. 
Let $M$ be an oriented compact three manifold and $\chi: H_{1}(M) \rightarrow \mathbb{C}^{*}$ be a character of finite order. For some $q \in \mathbb{N}^{*}$, the image of $\chi$ is contained a cyclic subgroup of order $q$ generated by $\alpha=e^{2 i \pi / q}$. As $\operatorname{Hom}\left(H_{1}(M), C_{q}\right)=$ $\left[M, B\left(C_{q}\right)\right]$, it follows that $\chi$ induces $q$-fold covering of $M$, denoted $\widetilde{M}$, with a canonical deck transformation. We will denote this transformation also by $\alpha$. If $\chi$ maps onto $C_{q}$, the canonical deck transformation sends $x$ to the other endpoint of the arc

that begins at $x$ and covers a loop representing an element of $(\chi)^{-1}(\alpha)$.

As the bordism group $\Omega_{3}\left(B\left(C_{q}\right)\right)=C_{q}$, we may conclude that $n$ disjoint copies of $M$, for some integer $n$, bounds bound a compact 4-manifold $W$ over $B\left(C_{q}\right)$. Note $n$ can be taken to be $q$. Let $\widetilde{W}$ be the induced covering with the deck transformation, denoted also by $\alpha$, that restricts to $\alpha$ on the boundary. This induces a $\mathbb{Z}\left[C_{q}\right]$ - module structure on $C_{*}(\widetilde{W})$, where the multiplication by $\alpha \in \mathbb{Z}\left[C_{q}\right]$ corresponds to the action of $\alpha$ on $\widetilde{W}$.

The cyclotomic field $\mathbb{Q}\left(C_{q}\right)$ is a natural $\mathbb{Z}\left[C_{q}\right]$-module and the twisted homology $H_{*}^{t}\left(W ; \mathbb{Q}\left(C_{q}\right)\right)$ is defined as the homology of

$$
C_{*}(\widetilde{W}) \otimes_{\mathbb{Z}\left[C_{q}\right]} \mathbb{Q}\left(C_{q}\right) .
$$

Since $\mathbb{Q}\left(C_{q}\right)$ is flat over $\mathbb{Z}\left[C_{q}\right]$, we get an isomorphism

$$
H_{*}^{t}\left(W ; \mathbb{Q}\left(C_{q}\right)\right) \simeq H_{*}(\widetilde{W}) \otimes_{\mathbb{Z}\left[C_{q}\right]} \mathbb{Q}\left(C_{q}\right) .
$$

Similarly, the twisted homology $H_{*}^{t}\left(M ; \mathbb{Q}\left(C_{q}\right)\right)$ is defined as the homology of

$$
C_{*}(\widetilde{M}) \otimes_{\mathbb{Z}\left[C_{q}\right]} \mathbb{Q}\left(C_{q}\right) .
$$

Let $\widetilde{\phi}$ be the intersection form on $H_{2}(\widetilde{W} ; \mathbb{Q})$ and define

$$
\phi_{\chi}(W): H_{2}^{t}\left(W ; \mathbb{Q}\left(C_{q}\right)\right) \times H_{2}^{t}\left(W ; \mathbb{Q}\left(C_{q}\right)\right) \rightarrow \mathbb{Q}\left(C_{q}\right)
$$

so that, for all $a, b$ in $\mathbb{Q}\left(C_{q}\right)$ and $x, y$ in $H_{2}(\widetilde{W})$,

$$
\phi_{\chi}(W)(x \otimes a, y \otimes b)=\bar{a} b \sum_{i=1}^{q} \widetilde{\phi}\left(x, \alpha^{i} y\right) \bar{\alpha}^{i},
$$

where $a \rightarrow \bar{a}$ denotes the involution on $\mathbb{Q}\left(C_{q}\right)$ induced by complex conjugation.

Definition 2.2 The Casson-Gordon $\sigma$-invariant of $(M, \chi)$ and the related nullity are

$$
\begin{gathered}
\sigma(M, \chi):=\frac{1}{n}\left(\operatorname{Sign}\left(\phi_{\chi}(W)\right)-\operatorname{Sign}(W)\right) \\
\eta(M, \chi):=\operatorname{dim} H_{1}^{t}\left(M ; \mathbb{Q}\left(C_{q}\right)\right) .
\end{gathered}
$$


If $U$ is a closed 4-manifold and $\chi: H_{1}(U) \rightarrow C_{q}$ we may define $\phi_{\chi}(U)$ as above. One has that modulo torsion the bordism group $\Omega_{4}\left(B\left(C_{q}\right)\right)$ is generated by the constant map from $C P(2)$ to $B\left(C_{q}\right)$. If $\chi$ is trivial, one has that $\operatorname{Sign}\left(\phi_{\chi}(U)\right)=\operatorname{Sign}(U)$. Since both signatures are invariant under cobordism, one has in general that $\operatorname{Sign}\left(\phi_{\chi}(U)\right)=\operatorname{Sign}(U)$. The independence of $\sigma(M, \chi)$ from the choice of $W$ and $n$ follows from this and Novikov additivity. One may see directly that these invariants do not depend on the choice of $q$. In this way Casson and Gordon argued that $\sigma(M, \chi)$ is an invariant. Alternatively one may use the Atiyah-Singer G-Signature theorem and Novikov additivity [AS].

We now describe a way to compute $\sigma(M, \chi)$ for a given surgery presentation of $(M, \chi)$.

Definition 2.3 Let $K$ be an oriented knot in $S^{3}$. Let $A$ be an embedded annulus such that $\partial A=K \cup K^{\prime}$ with $l k\left(K, K^{\prime}\right)=f$. A $p$-cable on $K$ with twist $f$ is defined to be the union of oriented parallel copies of $K$ lying in $A$ such that the number of copies with the same orientation minus the number with opposite orientation is equal to $p$.

Let us suppose that $M$ is obtained by surgery on a framed link $L=L_{1} \cup \cdots \cup L_{\mu}$ with framings $f_{1}, \ldots, f_{\mu}$. One shows that the linking matrix $\Lambda$ of $L$ with framings in the diagonal is a presentation matrix of $H_{1}(M)$ and a character on $H_{1}(M)$ is determined by $\alpha^{p_{i}}=\chi\left(m_{L_{i}}\right) \in C_{q}$ where $m_{L_{i}}$ denotes the class of the meridian of $L_{i}$. Let $\vec{p}=\left(p_{1}, \ldots, p_{\mu}\right)$. We use the following generalization of a formula in [CG2, Lemma (3.1)], where all $p_{i}$ are assumed to be 1 , that is given in $[\mathrm{Gi} 2$, Theorem(3.6)].

Proposition 2.4 Suppose $\chi$ maps onto $C_{q}$. Let $L^{\prime}$ with $\mu^{\prime}$ components be the link obtained from $L$ by replacing each component by a non-empty algebraic $p_{i}$-cable with twist $f_{i}$ along this component. Then, if $\lambda=e^{2 i r \pi / q}$, for $(r, q)=1$, one has

$$
\begin{gathered}
\sigma\left(M, \chi^{r}\right)=\sigma_{L^{\prime}}(\lambda)-\operatorname{Sign}(\Lambda)+2 \frac{r(q-r)}{q^{2}} \vec{p}^{\top} \Lambda \vec{p}, \\
\eta\left(M, \chi^{r}\right)=\eta_{L^{\prime}}(\lambda)-\mu^{\prime}+\mu .
\end{gathered}
$$

The following proposition collects some easy additivity properties of the $\sigma$ invariant and the nullity under the connected sum.

Proposition 2.5 Suppose that $M_{1}, M_{2}$ are connected. Then, for all $\chi_{i} \in$ $H^{1}\left(M_{i} ; C_{q}\right), i=1,2$, we have

$$
\sigma\left(M_{1} \# M_{2}, \chi_{1} \oplus \chi_{2}\right)=\sigma\left(M_{1}, \chi_{1}\right)+\sigma\left(M_{2}, \chi_{2}\right) .
$$


If both $\chi_{i}$ are non-trivial, then

$$
\eta\left(M_{1} \# M_{2}, \chi_{1} \oplus \chi_{2}\right)=\eta\left(M_{1}, \chi_{1}\right)+\eta\left(M_{2}, \chi_{2}\right)+1 .
$$

If one $\chi_{i}$ is trivial, then

$$
\eta\left(M_{1} \# M_{2}, \chi_{1} \oplus \chi_{2}\right)=\eta\left(M_{1}, \chi_{1}\right)+\eta\left(M_{2}, \chi_{2}\right) .
$$

Proposition 2.6 For all $\chi \in H_{1}\left(S^{1} \times S^{2} ; C_{q}\right)$, we have

$$
\begin{gathered}
\sigma\left(S^{1} \times S^{2}, \chi\right)=0 \\
\text { If } \chi \neq 0 \text {, then } \eta\left(S^{1} \times S^{2}, \chi\right)=0 \text {. If } \chi=0 \text {, then } \eta\left(S^{1} \times S^{2}, \chi\right)=1 .
\end{gathered}
$$

Proposition 2.6 for non-trivial $\chi$ can be proved for example by the use of Proposition 2.4, since $S^{1} \times S^{2}$ is obtained by surgery on the unknot framed 0 . However it is simplest to derive this result directly from the definitions.

\subsection{The Casson-Gordon $\tau$-invariant}

In this section, we recall the definition and some of the properties of the CassonGordon $\tau$-invariant. Let $C_{\infty}$ denote a multiplicative infinite cyclic group generated by $t$. For $\chi^{+}: H_{1}(M) \rightarrow C_{q} \oplus C_{\infty}$, we denote $\bar{\chi}: H_{1}(M) \rightarrow C_{q}$ the character obtained by composing $\chi^{+}$with projection on the first factor. The character $\chi^{+}$induces a $C_{q} \times C_{\infty}$-covering $\widetilde{M}_{\infty}$ of $M$.

Since the bordism group $\Omega_{3}\left(B\left(C_{q} \times C_{\infty}\right)\right)=C_{q}$, bounds a compact 4-manifold $W$ over $B\left(C_{q} \times C_{\infty}\right)$ Again $n$ can be taken from to be $q$.

If we identify $\mathbb{Z}\left[C_{q} \times C_{\infty}\right]$ with the Laurent polynomial ring $\mathbb{Z}\left[C_{q}\right]\left[t, t^{-1}\right]$, the field $\mathbb{Q}\left(C_{q}\right)(t)$ of rational functions over the cyclotomic field $\mathbb{Q}\left(C_{q}\right)$ is a flat $\mathbb{Z}\left[C_{q} \times C_{\infty}\right]$-module. We consider the chain complex $C_{*}\left(\widetilde{W}_{\infty}\right)$ as a $\mathbb{Z}\left[C_{q} \times C_{\infty}\right]$ module given by the deck transformation of the covering. Since $W$ is compact, the vector space $H_{2}^{t}\left(W ; \mathbb{Q}\left(C_{q}\right)(t)\right) \simeq H_{2}\left(\widetilde{W}_{\infty}\right) \otimes_{\mathbb{Z}\left[C_{q}\right]\left[t, t^{-1}\right]} \mathbb{Q}\left(C_{q}\right)(t)$ is finite dimensional.

We let $J$ denote the involution on $\mathbb{Q}\left(C_{q}\right)(t)$ that is linear over $\mathbb{Q}$ sends $t^{i}$ to $t^{-i}$ and $\alpha^{i}$ to $\alpha^{-i}$. As in [G], one defines a hermitian form, with respect to $J$,

$$
\phi_{\chi^{+}}: H_{2}^{t}\left(W ; \mathbb{Q}\left(C_{q}\right)(t)\right) \times H_{2}^{t}\left(W ; \mathbb{Q}\left(C_{q}\right)(t)\right) \rightarrow \mathbb{Q}\left(C_{q}\right)(t),
$$

such that

$$
\phi_{\chi^{+}}(x \otimes a, y \otimes b)=J(a) \cdot b \cdot \sum_{i \in \mathbb{Z}} \sum_{j=1}^{q} \widetilde{\phi^{+}}\left(x, t^{i} \alpha^{j} y\right) \bar{\alpha}^{j} t^{-i} .
$$


Here $\widetilde{\phi^{+}}$denotes the ordinary intersection form on $\widetilde{W}_{\infty}$. Let $\mathcal{W}\left(\mathbb{Q}\left(C_{q}\right)(t)\right)$ be the Witt group of non-singular hermitian forms on finite dimensional $\mathbb{Q}\left(C_{q}\right)(t)$ vector spaces. Let us consider $H_{2}^{t}\left(W ; \mathbb{Q}\left(C_{q}\right)(t)\right) /\left(\operatorname{Radical}\left(\phi_{\chi^{+}}\right)\right)$. The induced form on it represents an element in $\mathcal{W}\left(\mathbb{Q}\left(C_{q}\right)(t)\right)$, which we denote $w(W)$. Furthermore, the ordinary intersection form on $H_{2}(W ; \mathbb{Q})$ represents an element of $\mathcal{W}(\mathbb{Q})$. Let $w_{0}(W)$ be the image of this element in $\mathcal{W}\left(\mathbb{Q}\left(C_{q}\right)(t)\right)$.

Definition 2.7 The Casson-Gordon $\tau$-invariant of $\left(M, \chi^{+}\right)$is

$$
\tau\left(M, \chi^{+}\right):=\frac{1}{n}\left(w(W)-w_{0}(W)\right) \in \mathcal{W}\left(\mathbb{Q}\left(C_{q}\right)(t)\right) \otimes \mathbb{Q} .
$$

Suppose that $n M$ bounds another compact 4-manifold $W^{\prime}$ over $B\left(C_{q} \times C_{\infty}\right)$. Form the closed compact manifold over $B\left(C_{q} \times C_{\infty}\right), U:=W \cup W^{\prime}$ by gluing along the boundary. By Novikov additivity, we get $w(U)-w_{0}(U)=(w(W)-$ $\left.w_{0}(W)\right)-\left(w\left(W^{\prime}\right)-w_{0}\left(W^{\prime}\right)\right)$. Using $[\mathrm{CF}]$, the bordism group $\Omega_{4}\left(B\left(C_{q} \times C_{\infty}\right)\right)$, modulo torsion, is generated by $C P(2)$, with the constant map to $B\left(C_{q} \times C_{\infty}\right)$. We have that $w(C P(2))=w_{0}(C P(2))$. Since $w(U)$, and $w_{0}(U)$ only depend on the bordism class of $U$ over $B\left(C_{q} \times C_{\infty}\right)$, it follows that $w(U)=w_{0}(U)$ and $\tau\left(M, \chi^{+}\right)$is independent of the choice of $W$. Using the above techniques, one may check $\tau\left(M, \chi^{+}\right)$is independent of $n$.

If $A \in \mathcal{W}\left(\mathbb{Q}\left(C_{q}\right)(t)\right)$, let $A(t)$ be a matrix representative for $A$. The entries of $A(t)$ are Laurent polynomials with coefficients in $\mathbb{Q}\left(C_{q}\right)$. If $\lambda$ is in $S^{1} \subset \mathbb{C}$, then $A(\lambda)$ is hermitian and has a well defined signature $\sigma_{\lambda}(A)$. One can view $\sigma_{\lambda}(A)$ as a locally constant map on the complement of the set of the zeros of $\operatorname{det} A(\lambda)$. As in [CG1], we re-define $\sigma_{\lambda}(A)$ at each point of discontinuity as the average of the one-sided limits at the point.

We have the following estimate [Gi3, Equation (3.1)].

Proposition 2.8 Let $\chi^{+}: H_{1}(M) \rightarrow C_{q} \oplus C_{\infty}$ and $\bar{\chi}: H_{1}(M) \rightarrow C_{q}$ be $\chi^{+}$ followed by the projection to $C_{q}$. We have

$$
\left|\sigma_{1}\left(\tau\left(M, \chi^{+}\right)\right)-\sigma(M, \bar{\chi})\right| \leq \eta(M, \bar{\chi}) .
$$

\subsection{Linking forms}

Let $M$ be a rational homology 3 -sphere with linking form

$$
l: H_{1}(M) \times H_{1}(M) \rightarrow \mathbb{Q} / \mathbb{Z} .
$$

We have that $l$ is non-singular, that is the adjoint of $l$ is an isomorphism $\iota: H_{1}(M) \rightarrow \operatorname{Hom}\left(H_{1}(M), \mathbb{Q} / \mathbb{Z}\right)$. Let $H_{1}(M)^{*}$ denote $\operatorname{Hom}\left(H_{1}(M), \mathbb{C}^{*}\right)$. Let 
$\nu$ denote the map $\mathbb{Q} / \mathbb{Z} \rightarrow \mathbb{C}^{*}$ that sends $\frac{a}{b}$ to $e^{\frac{2 \pi i a}{b}}$. So we have an isomorphism

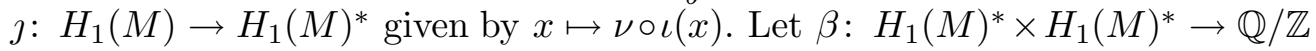
be the dual form defined by $\beta(\jmath x, \jmath y)=-l(x, y)$.

Definition 2.9 The form $\beta$ is metabolic with metabolizer $H$ if there exists a subgroup $H$ of $H_{1}(M)^{*}$ such that $H^{\perp}=H$.

Lemma 2.10 [Gi1] If $M$ bounds a spin 4-manifold $W$ then $\beta=\beta_{1} \oplus \beta_{2}$ where $\beta_{2}$ is metabolic and $\beta_{1}$ has an even presentation with rank dim $H_{2}(W ; \mathbb{Q})$ and signature $\operatorname{Sign}(W)$. Moreover, the set of characters that extend to $H_{1}(W)$ forms a metabolizer for $\beta_{2}$.

\subsection{Link invariants}

Let $L=L_{1} \cup \cdots \cup L_{\mu}$ be an oriented link in $S^{3}$. Let $N_{2}$ be the two-fold covering of $S^{3}$ branched along $L$ and $\beta_{L}$ be the linking form on $H_{1}\left(N_{2}\right)^{*}$, see previous section.

We suppose that the Alexander polynomial of $L$ satisfies

$$
\Delta_{L}(-1) \neq 0 .
$$

Hence, $N_{2}$ is a rational homology sphere. Note that if $\Delta_{L}(-1) \neq 1$, then $H_{1}\left(N_{2} ; \mathbb{Z}\right)$ is non-trivial.

Definition 2.11 For all characters $\chi$ in $H_{1}\left(N_{2}\right)^{*}$, the Casson-Gordon $\sigma$ invariant of $L$ and the related nullity are (see Definition 2.2):

$$
\begin{aligned}
\sigma(L, \chi) & :=\sigma\left(N_{2}, \chi\right), \\
\eta(L, \chi) & :=\eta\left(N_{2}, \chi\right) .
\end{aligned}
$$

Remark 2.12 If $L$ is a knot, then Definition 2.11 coincides with $\sigma(L, \chi)$ defined in [CG1, p.183]. 


\section{$3 \quad$ Framed link descriptions}

In this section, we study the Casson-Gordon $\tau$-invariants of the two-fold cover $M_{2}$ of the manifold $M_{0}$ described below.

Let $S^{3}-T(L)$ be the complement in $S^{3}$ of an open tubular neighborhood of $L$ in $S^{3}$ and $P$ be a planar surface with $\mu$ boundary components.

Let $S$ be a Seifert surface for $L$ and $\gamma_{i}$ for $i=1, \ldots, \mu$ be the curves where $S$ intersects the boundary of $S^{3}-T(L)$. We define $M_{0}$ as the result of gluing $P \times S^{1}$ to $S^{3}-T(L)$, where $P \times 1$ is glued along the curves $\gamma_{i}$. Let $*$ be a point in the boundary of $P$.

A recipe for drawing a framed link description for $M_{0}$ is given in the proof of Proposition 3.1.

\section{Proposition 3.1}

$$
H_{1}\left(M_{0}\right) \simeq \mathbb{Z} \oplus \mathbb{Z}^{\mu-1} \simeq\langle m\rangle \oplus \mathbb{Z}^{\mu-1},
$$

where $m$ denotes the class of $* \times S^{1}$ in $P \times S^{1}$.

Proof Form a 4-manifold $X$ by gluing $P \times D^{2}$ to $D^{4}$ along $S^{3}$ in such a way that the total framing on $L$ agrees with the Seifert surface $S$. The boundary of this 4-manifold is $M_{0}$. We can get a surgery description of $M_{0}$ in the following way: pick $\mu-1$ paths of $S$ joining up the components of $L$ in a chain. Deleting open neighborhoods of these paths in $S$ gives a Seifert surface for a knot $L^{\prime}$ obtained by doing a fusion of $L$ along bands that are neighborhoods of the original paths. Put a circle with a dot around each of these bands (representing a 4-dimensional 1-handle in Kirby's [K] notation), and the framing zero on $L^{\prime}$. This describes a handlebody decomposition of $X$.

One can then get a standard framed link description of $M_{0}$ by replacing the circle with dots with unknots $T_{1}, \ldots, T_{\mu-1}$ framed zero. This changes the 4 manifold but not the boundary. Note also that $l k\left(T_{i}, T_{j}\right)=0$ and $l k\left(T_{i}, L^{\prime}\right)=0$ for all $i=1, \ldots, \mu-1$. Hence $H_{1}\left(M_{0}\right) \simeq \mathbb{Z}^{\mu}$ and $m$ represents one of the generators.

We now consider an infinite cyclic covering $M_{\infty}$ of $M_{0}$, defined by a character $H_{1}\left(M_{0}\right) \rightarrow C_{\infty}=\langle t\rangle$ that sends $m$ to $t$ and the other generators to zero. Let us denote by $M_{2}$ the intermediate two-fold covering obtained by composing this character with the quotient map $C_{\infty} \rightarrow C_{2}$ sending $t$ to -1 . Let $m_{2}$ denote the loop in $M_{2}$ given by the inverse image of $m$. A recipe for drawing a framed link description for $M_{2}$ is given in the proof of Remark 3.3. 
Proposition 3.2 There is an isomorphism between $H_{1}\left(N_{2}\right)$ and the torsion subgroup of $H_{1}\left(M_{2}\right)$, which only depends on $L$. Moreover

$$
H_{1}\left(M_{2}\right) \simeq H_{1}\left(N_{2}\right) \oplus \mathbb{Z}^{\mu} \simeq H_{1}\left(N_{2}\right) \oplus\left\langle m_{2}\right\rangle \oplus \mathbb{Z}^{\mu-1} .
$$

Proof Let $R$ be the result of gluing $P \times D^{2}$ to $S^{3} \times I$ along $L \times 1 \subset S^{3} \times 1$ using the framing given by the Seifert surface. Thus $R$ is the result of adding $\mu-1$ 1-handles to $S^{3} \times I$ and then one 2-handle along $L^{\prime}$, as in the proof above. Then $X$ in the proof above can be obtained by gluing $D^{4}$ to $R$ along $S^{3} \times 0$. Since $D^{2}$ is the double branched cover of itself along the origin, $P \times D^{2}$ is the double branched cover of itself along $P \times 0$. Let $R_{2}$ denote the double branched cover of $R$ that is obtained by gluing $P \times D^{2}$ to $N_{2} \times I$ along a neighborhood of the lift of $L \times 1 \subset S^{3} \times 1$. We have that $\partial R_{2}=-N_{2} \sqcup M_{2}$, where $R_{2}$ is the result of adding $\mu-1$ 1-handles to $N_{2} \times I$ and then one 2-handle along the lift $L^{\prime}$. Moreover this lift of $L^{\prime}$ is null-homologous in $N_{2}$. It follows that $H_{1}\left(R_{2}\right)$ is isomorphic to $H_{1}\left(N_{2}\right) \oplus \mathbb{Z}^{\mu-1}$, with the inclusion of $N_{2}$ into $R_{2}$ inducing an isomorphism $i_{N}$ of $H_{1}\left(N_{2}\right)$ to the torsion subgroup of $H_{1}\left(R_{2}\right)$. Turning this handle decomposition upside down we have that $R_{2}$ is the result of adding to $M_{2} \times I$ one 2-handle along a neighborhood of $m_{2}$ and then $\mu-1$ 3-handles. It follows that $H_{1}\left(R_{2}\right) \oplus \mathbb{Z}=H_{1}\left(R_{2}\right) \oplus\left\langle m_{2}\right\rangle$ is isomorphic to $H_{1}\left(M_{2}\right)$ with the inclusion of $M_{2}$ in $R_{2}$ inducing an isomorphism $i_{M}$ of the torsion subgroup $H_{1}\left(M_{2}\right)$ to the torsion subgroup of $H_{1}\left(R_{2}\right)$. Thus $\left(i_{M}\right)^{-1} \circ i_{N}$ is an isomorphism from $H_{1}\left(N_{2}\right)$ to the torsion subgroup of $H_{1}\left(M_{2}\right)$ and this isomorphism is constructed without any arbitrary choices.

Remark 3.3 We could have proved Proposition 3.1 in a similar way to the proof of Proposition 3.2. We could have also proved Proposition 3.2 (except for the isomorphism only depending on $L$ ) in a similar way to the proof of Proposition 3.1 as follows. We can find a surgery description of $M_{2}$ from a surgery description of $N_{2}$. The procedure of how to visualize a lift of $L$ and the surface $S$ in $N_{2}$ is given in [AK]. One considers the lifts of the paths chosen in the proof of Proposition 3.1, on the lift of $S$. One then fuses the components of the lift of $L$ along these paths, obtaining a lift of $L^{\prime}$. The surgery description of $M_{2}$ is obtained by adding to the surgery description of $N_{2}$ the lift of $L^{\prime}$ with zero framing together with $\mu-1$ more unknotted zero-framed components encircling each fusion. The linking matrix of this link is a direct sum of that of $N_{2}$ and a $\mu \times \mu$ zero matrix. 
Let $i_{T}$ denote the inclusion of the torsion subgroup of $H_{1}\left(M_{2}\right)$ into $H_{1}\left(M_{2}\right)$, and let $\psi: H_{1}\left(N_{2}\right) \rightarrow H_{1}\left(M_{2}\right)$ denote the monomorphism given by $i_{T} \circ\left(i_{M}\right)^{-1} \circ$ $i_{N}$.

Theorem 3.4 Let $\chi^{+}: H_{1}\left(M_{2}\right) \rightarrow C_{q} \oplus C_{\infty}$. Let $\chi: H_{1}\left(N_{2}\right) \rightarrow C_{q}$ be $\chi^{+} \circ \psi$ composed with the projection to $C_{q}$. We have that:

$$
\left|\sigma_{1}\left(\tau\left(M_{2}, \chi^{+}\right)\right)-\sigma(L, \chi)\right| \leq \eta(L, \chi)+\mu .
$$

Remark 3.5 If $L$ is a knot, then $\tau\left(M_{2}, \chi^{+}\right)$coincides with $\tau(L, \chi)$ defined in [CG1, p.189].

Proof of Theorem 3.4 We use the surgery description of $M_{2}$ given in Remark 3.3. Let $P$ be given by the surgery description of $M_{2}$ but with the component corresponding to $L^{\prime}$ deleted. Hence,

$$
P=N_{2} \sharp(\mu-1) S^{1} \times S^{2} .
$$

$\chi+$ induces some character $\chi^{\prime}$ on $H_{1}(P)$.

According to Section 2.3, we let $\bar{\chi} \in H^{1}\left(M_{2} ; C_{q}\right)$ and $\bar{\chi}^{\prime} \in H^{1}\left(P ; C_{q}\right)$ denote the characters $\chi^{+}$and $\chi^{\prime}$ followed by the projection $C_{q} \oplus C_{\infty} \rightarrow C_{q}$. Using Propositions 2.5 and 2.6, one has that

$$
\sigma\left(P, \bar{\chi}^{\prime}\right)=\sigma(L, \chi) \text { and } \eta\left(P, \bar{\chi}^{\prime}\right)=\eta(L, \chi)+\mu-1 .
$$

Moreover, since $M_{2}$ is obtained by surgery on $L^{\prime}$ in $P$, it follows from [Gi3, Proposition (3.3)] that

$$
\begin{gathered}
\left|\sigma\left(P, \bar{\chi}^{\prime}\right)-\sigma\left(M_{2}, \bar{\chi}\right)\right|+\left|\eta\left(M_{2}, \bar{\chi}\right)-\eta\left(P, \bar{\chi}^{\prime}\right)\right| \leq 1 \text { or } \\
\left|\sigma(L, \chi)-\sigma\left(M_{2}, \bar{\chi}\right)\right|+\left|\eta\left(M_{2}, \bar{\chi}\right)-\eta(L, \chi)-\mu+1\right| \leq 1 .
\end{gathered}
$$

Thus

$$
\left|\sigma(L, \chi)-\sigma\left(M_{2}, \bar{\chi}\right)\right| \leq \eta(L, \chi)+\mu-\eta\left(M_{2}, \bar{\chi}\right) .
$$

Finally, one gets, by Theorem 2.8,

$$
\begin{gathered}
\left|\sigma_{1}\left(\tau\left(M_{2}, \chi^{+}\right)\right)-\sigma(L, \chi)\right| \leq\left|\sigma_{1}\left(\tau\left(M_{2}, \chi^{+}\right)\right)-\sigma\left(M_{2}, \bar{\chi}\right)\right|+\left|\sigma\left(M_{2}, \bar{\chi}\right)-\sigma(L, \chi)\right| \\
\leq \eta\left(M_{2}, \bar{\chi}\right)+\eta(L, \chi)+\mu-\eta\left(M_{2}, \bar{\chi}\right)=\eta(L, \chi)+\mu .
\end{gathered}
$$




\section{The slice genus of links}

See Section 2.5 for notations.

Theorem 4.1 Suppose $L$ is the boundary of a connected oriented properly embedded surface $F$ of genus $g$ in $B^{4}$, and that $\Delta_{L}(-1) \neq 0$. Then, $\beta_{L}$ can be written as a direct sum $\beta_{1} \oplus \beta_{2}$ such that the following two conditions hold:

1) $\beta_{1}$ has an even presentation of rank $2 g+\mu-1$ and signature $\sigma_{L}(-1)$, and $\beta_{2}$ is metabolic.

2) There is a metabolizer for $\beta_{2}$ such that for all characters $\chi$ of prime power order in this metabolizer,

$$
\left|\sigma(L, \chi)+\sigma_{L}(-1)\right| \leq \eta(L, \chi)+4 g+3 \mu-2 .
$$

Proof We let $b_{i}(X)$ denote the ith Betti number of a space $X$. We have $b_{1}(F)=2 g+\mu-1$.

Let $W_{0}^{\prime}$, with boundary $M_{0}^{\prime}$, be the complement of an open tubular neighborhood of $F$ in $B^{4}$. By the Thom isomorphism, excision, and the long exact sequence of the pair $\left(B^{4}, W_{0}^{\prime}\right), W_{0}^{\prime}$ has the homology of $S^{1}$ wedge $b_{1}(F) 2$ spheres. Let $W_{2}^{\prime}$ with boundary $M_{2}^{\prime}$ be the two-fold covering of $W_{0}^{\prime}$. Note that if $F$ is planar, $M_{0}^{\prime}=M_{0}$, and $M_{2}^{\prime}=M_{2}$ (see Section 3).

Let $V_{2}$ be the two-fold covering of $B^{4}$ with branched set $F$. Note that $V_{2}$ is spin as $w_{2}\left(V_{2}\right)$ is the pull-up of a class in $H^{2}\left(B^{4}, \mathbb{Z}_{2}\right)$, by [Gi5, Theorem 7], for instance. The boundary of $V_{2}$ is $N_{2}$. As in [Gi1], one calculates that $b_{2}\left(V_{2}\right)=2 g+\mu-1$. One has $\operatorname{Sign}\left(V_{2}\right)=\sigma_{L}(-1)$ by $[\mathrm{V}]$.

By Lemma 2.10, $\beta_{L}$ can be written as a direct sum $\beta_{1} \oplus \beta_{2}$ as in condition 1) above, such that the characters on $H_{1}\left(N_{2}\right)$ that extend to $H_{1}\left(V_{2}\right)$ form a metabolizer $H$ for $\beta_{2}$. We now suppose $\chi \in H$ and show that Condition 2) holds for $\chi$.

We also let $\chi$ denote an extension of $\chi$ to $H_{1}\left(V_{2}\right)$ with image some cyclic group $C_{q}$ where $q$ is a power of a prime integer (possibly larger than those corresponding to the character on $\left.H_{1}\left(N_{2}\right)\right)$. Of course $\chi \in H^{1}\left(V_{2}, C_{q}\right)$ restricted to $W_{2}^{\prime}$ extends $\chi$ restricted to $M_{2}^{\prime}$. We simply denote all these restrictions by $\chi$.

Let $W_{\infty}^{\prime}$ denote the infinite cyclic cover of $W_{0}^{\prime}$. Note that $W_{2}^{\prime}$ is a quotient of this covering space. $\chi$ induces a $C_{q}$-covering of $V_{2}$ and thus of $W_{2}^{\prime}$. If we pull the $C_{q}$-covering of $W_{2}^{\prime}$ up to $W_{\infty}^{\prime}$, we obtain $\widetilde{W}_{\infty}^{\prime}$, a $C_{q} \times C_{\infty}$-covering of $W_{2}^{\prime}$. If we identify properly $F \times S^{1}$ in $M_{2}^{\prime}$, this covering restricted to $F \times S^{1}$ is given by 
a character $H_{1}\left(F \times S^{1}\right) \simeq H_{1}(F) \oplus H_{1}\left(S^{1}\right) \rightarrow C_{q} \times C_{\infty}$ that maps $H_{1}(F)$ to zero in $C_{\infty}, H_{1}\left(S^{1}\right)$ to zero in $C_{q}$ and isomorphically onto $C_{\infty}$. For this note: since $\operatorname{Hom}\left(H_{1}(F), \mathbb{Z}\right)=H^{1}(F)=\left[F, S^{1}\right]$, we may define diffeomorphisms of $F \times S^{1}$ that induce the identity on the second factor of $H_{1}\left(F \times S^{1}\right) \approx H_{1}(F) \oplus \mathbb{Z}$, and send $(x, 0) \in H_{1}(F) \oplus \mathbb{Z}$, to $(x, f(x)) \in H_{1}(F) \oplus \mathbb{Z}$, for any $f \in \operatorname{Hom}\left(H_{1}(F), \mathbb{Z}\right)$.

As in [Gi1], choose inductively a collection of $g$ disjoint curves in the kernel of $\chi$ that form a metabolizer for the intersection form on $H_{1}(F) / H_{1}(\partial F)$. By taking a tubular neighborhood of these curves in $F$, we obtain a collection of $S^{1} \times I$ embedded in $F$. Using these embeddings we can attach round 2-handles $\left(B^{2} \times I\right) \times S^{1}$ along $\left(S^{1} \times I\right) \times S^{1}$ to the trivial cobordism $M_{2}^{\prime} \times I$ and obtain a cobordism $\Omega$ between $M_{2}$ and $M_{2}^{\prime}$.

Let $U=W_{2}^{\prime} \cup_{M_{2}^{\prime}} \Omega$ with boundary $M_{2}$. The $C_{q} \times C_{\infty}$-covering of $W_{2}^{\prime}$ extends uniquely to $U$. Note that $\Omega$ may also be viewed as the result of attaching round 1-handles to $M_{2} \times I$.

As in [Gi1], $\operatorname{Sign}\left(W_{2}^{\prime}\right)=\operatorname{Sign}\left(V_{2}\right)$. Since the intersection form on $\Omega$ is zero, we get $\operatorname{Sign}(U)=\operatorname{Sign}\left(W_{2}^{\prime}\right)=\operatorname{Sign}\left(V_{2}\right)=\sigma_{L}(-1)$. The $C_{q} \times C_{\infty}$-covering of $\Omega$, restricted to each round 2-handle is $q$ copies of $B^{2} \times I \times \mathbb{R}$ attached to the trivial cobordism $\widetilde{M}_{\infty}^{\prime} \times I$ along $q$ copies of $S^{1} \times I \times \mathbb{R}$. Using a Mayer-Vietoris sequence, one sees that the inclusion induces an isomorphism (which preserves the Hermitian form)

$$
H_{2}^{t}\left(U ; \mathbb{Q}\left(C_{q}\right)(t)\right) \simeq H_{2}^{t}\left(W_{2}^{\prime} ; \mathbb{Q}\left(C_{q}\right)(t)\right) .
$$

Thus, if $w\left(W_{2}^{\prime}\right)$ denotes the image of the intersection form on $H_{2}^{t}\left(W_{2}^{\prime} ; \mathbb{Q}\left(C_{q}\right)(t)\right)$ in $\mathcal{W}\left(\mathbb{Q}\left(C_{q}\right)(t)\right)$, we get $\sigma_{1}\left(\tau\left(M_{2}, \chi^{+}\right)\right)=\sigma_{1}\left(w\left(W_{2}^{\prime}\right)\right)-\sigma_{L}(-1)$.

If $q$ is a prime power, we may apply Lemma 2 of [Gi1] and conclude that $H_{i}\left(\widetilde{W}_{\infty}^{\prime} ; \mathbb{Q}\right)$ is finite dimensional for all $i \neq 2$. Thus, $H_{i}^{t}\left(W_{2}^{\prime} ; \mathbb{Q}\left(C_{q}\right)(t)\right)$ is zero for all $i \neq 2$. Since the Euler characteristic of $W_{2}^{\prime}$ with coefficients in $\mathbb{Q}\left(C_{q}\right)(t)$ coincides with those with coefficients in $\mathbb{Q}$, we get $\operatorname{dim} H_{2}^{t}\left(W_{2}^{\prime} ; \mathbb{Q}\left(C_{q}\right)(t)\right)=$ $\chi\left(W_{2}^{\prime}\right)=2 \chi\left(W_{0}^{\prime}\right)=2(1-\chi(F))=2 b_{1}(F)$. Thus $\mid \sigma_{1}\left(\tau\left(M_{2}, \chi^{+}\right)+\sigma_{L}(-1) \mid \leq\right.$ $2 b_{1}(F)$. Hence,

$$
\begin{aligned}
& \left|\sigma(L, \chi)+\sigma_{L}(-1)\right| \leq\left|\sigma(L, \chi)-\sigma_{1}\left(\tau\left(M_{2}, \chi\right)^{+}\right)\right|+\left|\sigma_{1}\left(\tau\left(M_{2}, \chi\right)^{+}\right)+\sigma_{L}(-1)\right| \\
& \leq \eta(L, \chi)+\mu+2(2 g+\mu-1)=\eta(L, \chi)+4 g+3 \mu-2 \text { by Theorem 3.4. }
\end{aligned}
$$

\section{$5 \quad$ Examples}

Let $L=L_{1} \cup L_{2}$ be the link with two components of Figure 1 and $S$ be the Seifert surface of $L$ given by the picture. The squares with $K$ denote two 
parallel copies with linking number 0 of an arc tied in the knot $K$. Note that $L$ is actually a family of examples. Specific links are determined by the choice of two parameters: a knot $K$ and a positive integer $h$. Since $S$ has genus $h$, the slice genus of $L$ is at most $h$.

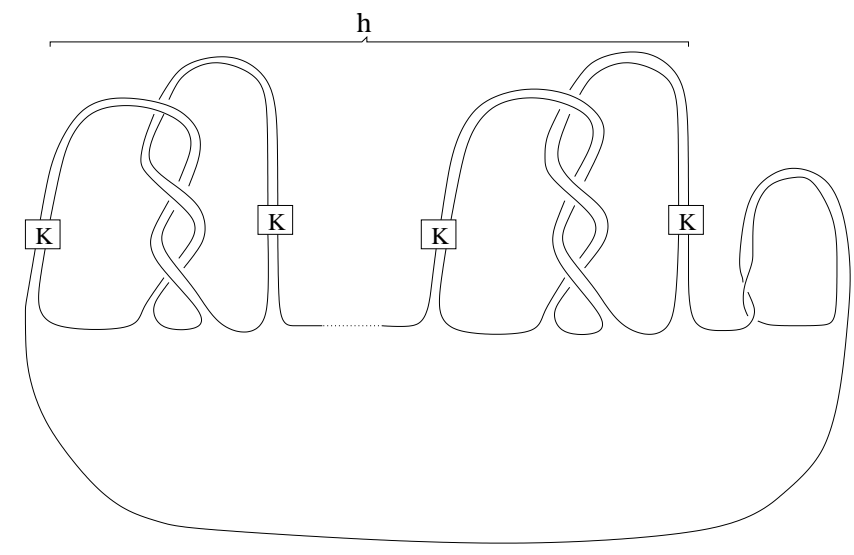

Figure 1: The link $L$

One calculates that $\sigma_{L}(\lambda)=1$, and $n_{L}(\lambda)=0$ for all $\lambda$. Thus, the MurasugiTristram inequality says nothing about the slice genus of $L$. In fact, if $K$ is a slice knot, then one can surger this surface to obtain a smooth cylinder in the 4 -ball with boundary $L$. Thus there can be no arguments based solely on a Seifert pairing for $L$ that would imply that the slice genus is non-zero.

Theorem 5.1 If $\sigma_{K}\left(e^{2 i \pi / 3}\right) \geq 2 h$ or $\sigma_{K}\left(e^{2 i \pi / 3}\right) \leq-2 h-2$, then $L$ has slice genus $h$.

Proof Using [AK], a surgery presentation of $N_{2}$ as surgery on a framed link of $2 h+1$ components can be obtained from the surface $S$ (see Figure 2).

Let $Q$ be the 3-manifold obtained from the link pictured in Figure 2. Here $K^{\prime}$ denotes $K$ with the string orientation reversed. Since $R P(3)$ is obtained by surgery on the unknot framed 2 , we get:

$$
N_{2}=R P(3) \#{ }_{h} Q .
$$

The linking matrix of the framed link of the surgery presentation of $N_{2}$ is $\Lambda=[2] \oplus \oplus^{h}\left[\begin{array}{ll}0 & 3 \\ 3 & 0\end{array}\right] . \Lambda$ is a presentation matrix of $\left(H_{1}\left(N_{2}\right)^{*}, \beta_{L}\right)$; we obtain

$$
H_{1}\left(N_{2}\right)^{*} \simeq \mathbb{Z}_{2} \bigoplus \oplus^{2 h} \mathbb{Z}_{3}
$$




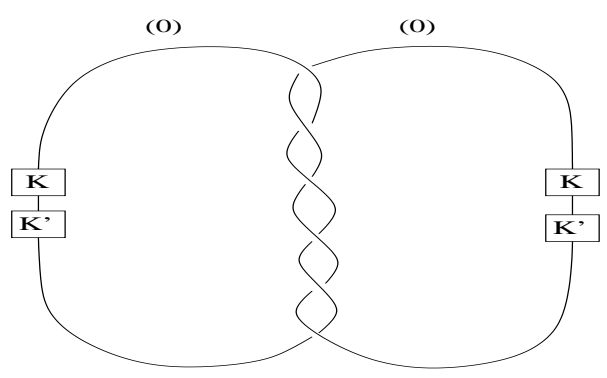

Figure 2: Surgery presentation of $Q$

and $\beta_{L}$ is given by the following matrix, with entries in $\mathbb{Q} / \mathbb{Z}$ :

$$
[1 / 2] \bigoplus \oplus^{h}\left[\begin{array}{cc}
0 & 1 / 3 \\
1 / 3 & 0
\end{array}\right]
$$

By Theorem 4.1, if $L$ bounds a surface of genus $h-1$ in $B^{4}$, then $\beta_{L}$ must be decomposed as $\beta_{1} \oplus \beta_{2}$ where:

1) $\beta_{1}$ has an even presentation matrix of rank $2 h-1$, and signature 1 (all we really need here is that it has a rank $2 h-1$ presentation.)

2) $\beta_{2}$ is metabolic and for all characters $\chi$ of prime power order in some metabolizer of $\beta_{2}$, the following inequality holds:

$$
|\sigma(L, \chi)+1|-\eta(L, \chi) \leq 4 h .
$$

As $\mathbb{Z}_{2} \oplus \oplus^{2 h} \mathbb{Z}_{3}$ does not have a rank $2 h-1$ presentation, $\beta_{2}$ is non-trivial. As metabolic forms are defined on groups whose cardinality is a square, $\beta_{2}$ is defined on a group with no 2 -torsion. Thus the metabolizer contains a nontrivial character of order three satisfying $\beta_{L}(\chi, \chi)=0$.

The first homology of $Q$ is $\mathbb{Z}_{3} \oplus \mathbb{Z}_{3}$, generated by, say, $m_{1}$ and $m_{2}$, positive meridians of these components. Each of these components is oriented counterclockwise. We first work out $\sigma(Q, \chi)$ and $\eta(Q, \chi)$ for characters of order three. Let $\chi_{\left(a_{1}, a_{2}\right)}$ denote the character on $H_{1}(Q)$ sending $m_{j}$ to $e^{\frac{2 i \pi a_{j}}{3}}$, where the $a_{j}$ take the values zero and \pm 1 .

We use Proposition 2.4 to compute $\sigma\left(Q, \chi_{(1,0)}\right)$ and $\eta\left(Q, \chi_{(1,0)}\right)$ assuming that $K$ is trivial. For this, one may adapt the trick illustrated on a link with 2 twists between the components [Gi2, Fig (3.3), Remark (3.65b)]. In the case $K$ is the unknot, we obtain

$$
\sigma\left(Q, \chi_{(1,0)}\right)=1 \quad \text { and } \quad \eta\left(Q, \chi_{(1,0)}\right)=0 .
$$


It is not difficult to see that inserting the knots of the type $K$ changes the result as follows (note that $K$ and $K^{\prime}$ have the same Tristram-Levine signatures):

$$
\sigma\left(Q, \chi_{(1,0)}\right)=1+2 \sigma_{K}\left(e^{2 \pi i / 3}\right) \quad \text { and } \quad \eta\left(Q, \chi_{(1,0)}\right)=0 .
$$

These same values hold for the characters $\chi_{(-1,0)}$ and $\chi_{(0, \pm 1)}$ by symmetry.

Using Proposition 2.4

$$
\begin{array}{cl}
\sigma\left(Q, \pm \chi_{(1,1)}\right)=-1-24 / 9+4 \sigma_{K}\left(e^{2 \pi i / 3}\right), & \eta\left(Q, \pm \chi_{(1,1)}\right)=0 \\
\sigma\left(Q, \pm \chi_{(1,-1)}\right)=4+24 / 9+4 \sigma_{K}\left(e^{2 \pi i / 3}\right) \quad \text { and } & \eta\left(Q, \pm \chi_{(1,-1)}\right)=1 .
\end{array}
$$

One also has

$$
\sigma\left(Q, \chi_{(0,0)}\right)=0 \quad \text { and } \quad \eta\left(Q, \chi_{(0,0)}\right)=0 .
$$

Any order three character on $N_{2}$ that is self annihilating under the linking form is given as the sum of the trivial character on $R P(3)$ and characters of type $\chi_{(0,0)}, \chi_{( \pm 1,0)}$ and $\chi_{(0, \pm 1)}$ on $Q$ and characters of type $\pm \chi_{(1,1)}+ \pm \chi_{(1,-1)}$ on $Q \# Q$. Using Proposition 2.5, one can calculate $\sigma(L, \chi)$ and $\eta(L, \chi)$ for all these characters $\chi$. It is now a trivial matter to check that for every non-trivial character with $\beta(\chi, \chi)=0$, the inequality $(*)$ is not satisfied.

\section{References}

[AK] Akbulut, S., Kirby, R., Branched covers of surfaces in 4-manifolds, Math. Ann. 252, 111-131 (1980).

[AS] Atiyah, M. F., Singer, I. M., The index of elliptic operators. III, Ann. of Math. (2) 87, 546-604 (1968).

[CF] Conner, P. E., Floyd, E.E., Differential Periodic Maps, Ergebnisse der Mathematik und ihrer Grenzgebiete, 33, Springer-Verlag, (1964).

[CG1] Casson, A. J., Gordon, C. Mc A., Cobordism of classical knots, Progr. Math., 62, A La Recherche de la Topologie Perdue, Birkhauser, Boston, MA, 181-199 (1986).

[CG2] Casson, A. J., Gordon, C. Mc A., On slice knots in dimension three, Proc. Symp. in Pure Math. XXX, 2, 39-53 (1978).

[Gi1] Gilmer, P. M., On the slice genus of knots, Invent. Math. 66, 191-197 (1982).

[Gi2] Gilmer, P. M., Configurations of surfaces in 4-manifolds, Trans. Amer. Math. Soc. 264, 353-380 (1981).

[Gi3] Gilmer, P. M., Slice knots in $S^{3}$, Quart. J. Math. Oxford 34, 305-322 (1983).

[Gi4] Gilmer, P. M., Classical knot and link concordance, Comment. Math. Helv. 68, 1-19 (1993).

Algebraic 83 Geometric Topology, Volume 3 (2003) 
[Gi5] Gilmer, P. M., Signatures of singular branched covers, Math. Ann. 295 (4), 643-659 (1993).

[GL] Gilmer, P. M., Livingston, C., The Casson-Gordon invariant and link concordance, Topology 31 , (3), 475-492 (1992).

[G] Gordon, C. McA., Some aspects of classical knot theory, Knot theory (Proc. Sem., Plans-sur-Bex, 1977), Lecture Notes in Math., 685, Springer Verlag, Berlin, 1-60 (1978).

[K] Kirby, R. C., The Topology of 4-manifolds, Lecture Notes in Math 1374 Springer Verlag, Berlin (1989).

[Le] Levine, J., Knot cobordism groups in codimension two, Comment. Math. Helv. 44 229-244 (1969).

[L] Lines D., Cobordisme de noeuds fibrés et de leur monodromie, Knots, braids and singularities (Plans-sur-Bex, 1982), 147-173, Monogr. Enseign. Math., 31, Enseignement Math., Geneva, (1983).

[Li] Litherland, R. A., Cobordism of satellite knots, Four-Manifold Theory (Durham, N.H., 1982), Contemp. Math., 35, Amer. Math. Soc., Providence, RI, 327-362 (1984).

[M] Murasugi, K., On a certain numerical invariant of link types, Trans. Amer. Math. Soc. 117 , 387-422 (1965).

[N] Naik, S. Casson-Gordon invariants of genus one knots and concordance to reverses, J. Knot Theory Ramifications 5, 661-677 (1996).

[T] Tristram, A. G., Some cobordism invariants for links, Proc, Camb. Philos. Soc., 66, 251-264 (1969).

[V] Viro, O. Ja. Branched coverings of manifolds with boundary, and invariants of links. I, Math. USSR-Izv. 7 1239-1256 (1973).

Laboratoire I.R.M.A. Université Louis Pasteur

Strasbourg, France

and

Department of Mathematics, Louisiana State University

Baton Rouge, LA 70803, USA

Email: vincent.florens@irma.u-strasbg.fr and gilmer@math.lsu.edu 